\title{
An Experimental Study of Wave Runup: Cylinder Fixed in Waves versus Cylinder Surging in Still Water
}

\author{
Deping Cao, Edmond Lo Yat-Man, Wei Jian, and Zhenhua Huang
}

\begin{abstract}
Many previous studies have been reported for the wave runup on fixed cylinders but few has been done on surging cylinders. Physically, there is conversion of the kinetic energy of water particles into potential energy during the runup on a fixed cylinder. Several formulas have been reported to correlate the runup with the velocity head. Whether this holds for a surging cylinder or not remains unknown. In the present study, experiments are conducted to investigate the relationship between runup on a cylinder and the relative velocity between the cylinder and the water particles around it. Both runup on a fixed cylinder due to an incident wave and that on surging cylinders due to the surge motion are studied. The results show that for both cases an increase in velocity head leads to an increase in runup.
\end{abstract}

Index Terms-Fixed cylinder, runup, surging cylinder, velocity head.

\section{INTRODUCTION}

Water wave runup is the vertical up-rush of water when an incident wave impinges upon a free surface penetrating body. The offshore industry is particularly interested in the accurate prediction of the maximum runup height for which significant volumes of water can be projected above mean sea level. When an incident wave hits a surface penetrating structure, the wave undergoes a violent transformation where some portion of the momentum of incident wave is directed vertically upward. To conserve energy, this momentum flux results in a rapid amplification of the waveform at the free-surface-body interface.

The wave runup on the forward vertical legs of both fixed and floating offshore structures is of particular interest to platform designers. In harsh ocean environments, the amplification of the incident wave may give rise to pressure impulse loads on the underside of the deck structure, referred to as a slamming force in the offshore structure community. In the instance of wave runup, a pressure impulse event occurs when a horizontal element, such as the platform deck or a body suspended from it, is impacted by a discrete volume

Manuscript received November 12, 2015; revised January 20, 2016. This work was supported by the Singapore Maritime Institute (Deepwater Technology R\&D Programme).

Deping Cao and Wei Jian are with Maritime Institute at Nanyang Technological University, Singapore 639798 (e-mail: DPCAO@ntu.edu.sg, jianwei@ntu.edu.sg).

Edmond Lo Yat-Man is with School of Civil and Environmental Engineering, Nanyang Technological University, Singapore 639798 (e-mail: cymlo@ntu.edu.sg).

Zhenhua Huang is with the Department of Ocean and Resources Engineering, School of Ocean and Earth Science and Technology, University of Hawaii at Manoa, Honolulu, Hawaii 96822-2303, USA (e-mail zhenhua@hawaii.edu). of water rushing up the weather side of a platform column. While not posing a threat to the overall structural integrity of the platform, wave runup is generally associated with localized structural damage. The accurate estimation of wave runup, and hence the air-gap, is thus extremely important for mitigating the hazards associated with pressure impulse events.

TABLE I: FORMULAS USED IN PREVIOUS STUDIES

\begin{tabular}{|c|c|c|}
\hline Source & $\begin{array}{l}\text { Surging/ } \\
\text { fixed }\end{array}$ & Formulas used \\
\hline [1] & Fixed & $\begin{array}{l}R_{u}=\eta_{\max }+\frac{u^{2}}{2 g} \\
\text { where } R_{u} \text { is the wave runup, } \eta_{\max } \text { is the maximum } \\
\text { wave crest and the velocity and } g \text { is the gravity } \\
\text { acceleration, } u \text { at } \eta_{\max } \text { was computed using } \\
\text { McCowan's solitary wave theory }\end{array}$ \\
\hline [2] & Fixed & N/A \\
\hline [3] & Fixed & $\begin{array}{l}\text { Same as Hallermeier (1976) except that } u \text { at } \eta_{\max } \\
\text { was computed using Dean's fifth-order stream } \\
\text { function wave theory }\end{array}$ \\
\hline [4] & Fixed & $\begin{array}{l}\text { Linear theory, Stokes second order, third order } \\
\text { theories }\end{array}$ \\
\hline
\end{tabular}

[5] Fixed

$R_{u}=\frac{H}{2}+7.43 \frac{u^{2}}{2 g}$ [6] Fixed $\quad R_{u}=0.56 H+6.52 \frac{u^{2}}{2 g}$

where $H$ is the incident wave height, $u$ was the wave crest velocity obtained from stream-function

1. Linear diffraction theory; 2. Kriebel's superposition theory using two Fourier components; 3. Velocity head method using linear [7] Fixed wave theory for the crest kinematics; 4 . Velocity head method using a high-order wave theory for the crest kinematics; 5 . The same method as Niedzwecki and Huston (1992)

$R_{u, \max }=\eta_{\max }+\frac{u_{\max }^{2}}{2 g}$

[8] Fixed

where $u_{\max }$ is the maximum water particle velocity at $\eta_{\max }$, which is calculated using second order Stokes theory

$$
R_{u, 2 \%}=\eta_{\max , 2 \%}+m \frac{u_{2 \%}^{2}}{2 g}
$$

[9] Fixed

where $\eta_{\max , 2 \%}$ is the crest level of the $2 \%$ highest wave, $u_{2} \%$ is the horizontal particle velocity in the top of the crest for the same wave; $m$ was a function of peak wave steepness; stream function theory was used to calculate $\eta_{\max , 2 \%}$ and $u_{2 \%}$ $R_{u, 2 \%}=\eta_{\max , 2 \%}+m \frac{u_{2 \%}^{2}}{2 g} R_{u, \max }=\eta_{\max }+m \frac{u_{\max }^{2}}{2 g}$

[10] Fixed $\quad m$ was a function of peak wave steepness; stream function theory was used to calculate $\eta_{\max }, \eta_{2} \%$, $u_{\max }$ and $u_{2 \%}$

Many previous studies [1]-[10] have been reported on wave runup on fixed columns. Most of these studies further 
developed equations by empirical fits to experimental data involving both the velocity head and incident wave height, as summarized in Table I.

Floating offshore structures are generally not completely fixed and how the motion of the structure influences the wave runup has seldom been reported. Intuitively, one may envisage that the wave runup should be correlated with the relative velocity when surging motion is involved. In the present study, two different scenarios are studied. One is wave runup on a fixed cylinder and the other is runup on a cylinder surging in still water in a sinusoidal way. Results are analyzed and compared to study the relationship between the runup and the relative velocity head.

\section{EXPERIMENTAL SETUP}

\section{A. Experimental Setup}

The experiments were conducted in a wave flume at the Hydraulics Modelling Laboratory, Nanyang Technological University. The flume has dimensions of $44.5 \mathrm{~m}$ long, $1.55 \mathrm{~m}$ wide and $1.5 \mathrm{~m}$ deep, as sketched in Fig. 1.

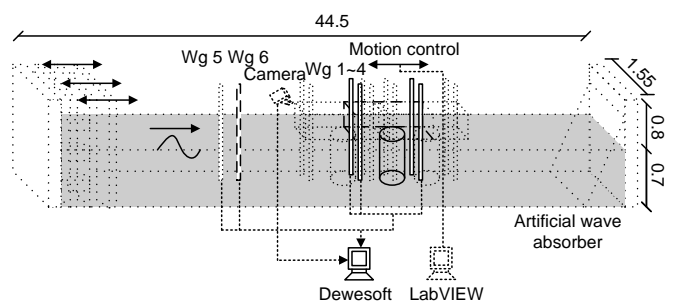

(a)

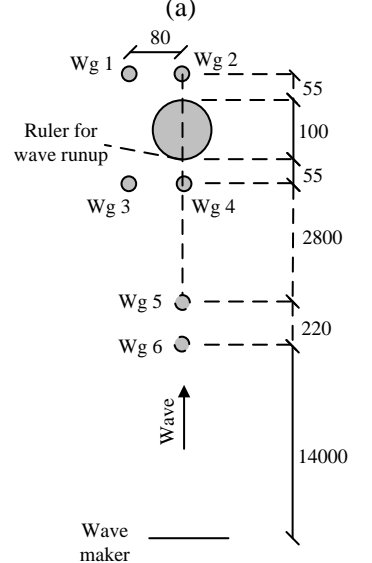

(b)

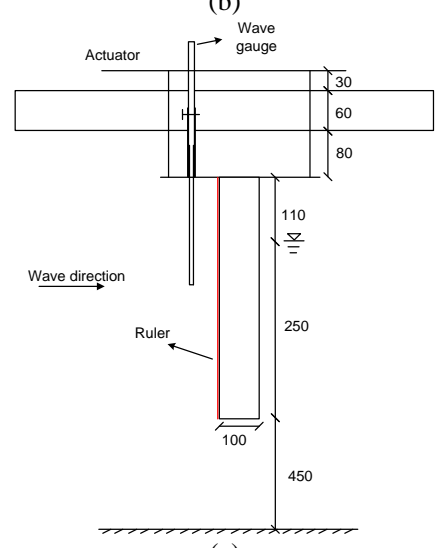

(c)

Fig. 1. Schematic drawing of (a) wave flume setup; (b) detailed locations of wave gauges $(\mathrm{Wg})$; (c) cylinder model. (Note units for (a) are $\mathrm{m}$, and for (b) and (c), mm).
Incident waves are generated by a two dimensional wave generator that is controlled by a DHI active wave absorption control system at the upstream end of the flume while an artificial wave absorber is installed at the downstream. A platform driven in surge by an actuator is located at a distance of $17 \mathrm{~m}$ from the wave generator. A cylinder model (see Fig. $1 \mathrm{c}$ for the sketch of the model) with diameter $=10 \mathrm{~cm}$ and length $=36 \mathrm{~cm}$ is attached to the platform. A total of $6 \mathrm{HR}$ Wallingford resistance type wave gauges are installed, two at 2.8 and $3.02 \mathrm{~m}$ in front of the cylinder respectively, to measure the incident waves and four mounted around the cylinder model to characterize the wave elevation (see Fig. $1 \mathrm{~b}$ for detailed locations of the gauges). A high speed camera (DS-CAM-300) is used to capture the wave runup via images of a measurement tape glued onto the front face of the cylinder. The camera records at a frame rate of $60 \mathrm{f} / \mathrm{s}$ resulting in the accuracy of video pictures being up to $0.5 \mathrm{~mm}$. An Ultralab sensor (ULS 40D) is used to record the surge motion of the actuator-driven platform and cylinder with a resolution of $1 \mathrm{~mm}$ achieved.

A Dewesoft data acquisition system is used to: (1) synchronize the camera and the wave gauges and (2) collect the wave elevation and wave runup data. LabVIEW programing is used to prescribe a sinusoidal signal for the actuator surge.

\section{B. Experimental Conditions}

Two different scenarios are experimentally tested. One is to study the runup of regular waves on a fixed cylinder with wave conditions shown in Table II (specifically F1-F6). The other is to investigate the runup on a surging cylinder due to the surge motion at the same frequencies as F1-F6 (see Table II, S1-S6 for the amplitude of surge motion). The water depth is kept constant at $d=0.7 \mathrm{~m}$. Note that in order to have a direct comparison between runup on fixed cylinder in wave and cylinder surging in still water for the same frequency, the surge amplitudes are set to be the values in Table II so that the velocity of the surge motion is close to that of maximum water particle velocity calculated using linear theory for the corresponding case of fixed cylinder in wave.

TABLE II: EXPERIMENTAL CONDITIONS FOR FIXED (F1-F6) AND SURGING (S1-S6) CYLINDERS

\begin{tabular}{lll}
\hline \hline Test & $\begin{array}{l}\text { Wave amplitude, } A_{w}(\mathrm{~cm}) \text { or } \\
\text { Amplitude of surge motion, } A_{s}\end{array}$ & $\begin{array}{l}\text { Wave period, } T_{w}(\mathrm{~s}) \text { or } \\
\text { Period of surge motion, } \\
T_{s}(\mathrm{~s})\end{array}$ \\
No. & $(\mathrm{cm})$ & 0.9 \\
\hline F1 & 2.16 & 1.2 \\
F2 & 2.49 & 1.5 \\
F3 & 2.38 & 1.8 \\
F4 & 2.29 & 2 \\
F5 & 2.35 & 2 \\
F6 & 3.53 & 0.9 \\
S1 & 2.33 & 1.2 \\
S2 & 2.52 & 1.5 \\
S3 & 2.53 & 1.8 \\
S4 & 2.67 & 2 \\
S5 & 2.92 & 2 \\
S6 & 4.40 & \\
\hline \hline
\end{tabular}

\section{Data Analysis}

Since all the frequencies used in the study is less than $2 \mathrm{~Hz}$ (see Table II), in order to eliminate higher frequency 
components, a low pass filter with a cut-off frequency of 5 $\mathrm{Hz}$ is used to filter all the original recorded signals while retaining some harmonics. For fixed cylinder in waves, Fast Fourier Transform (FFT) is applied to a time window of 10 wave periods for the most stable waves for all 6 wave gauges in order to obtain the fundamental amplitude and frequency. Goda and Suzuki's method [11] is used to do wave separation for signals from wave gauges 5 and 6 to obtain the incident wave amplitude. For the cylinder surging in still water, the fundamental amplitude and frequency of the motion are similarly obtained via FFT on the most stable section of the recorded trajectory signals. The surge velocity of the cylinder is then calculated from the measured surge displacement. In all tests, the corresponding sections of the videos recorded by the high speed camera are digitized to obtain the runup on the cylinders.

\section{RESUlTS AND DISCUSSION}

A sample of the time series of surface elevations of wave gauges 5 and 6 for Test F1 is shown in Fig. 2a. The most stable sections of ten cycles $(9 \mathrm{~s}$, the section in the dotted frame) of wave signals are chosen for FFT (the result is shown in Fig. 2b) and then wave separation is conducted to obtain the incident wave amplitude using Goda and Suzuki's method [11]. The corresponding video recorded during this time section is digitized to obtain the wave runup. The digitized runup on the fixed cylinder is almost constant for the 10 cycles analyzed as the incident wave is quite stable.

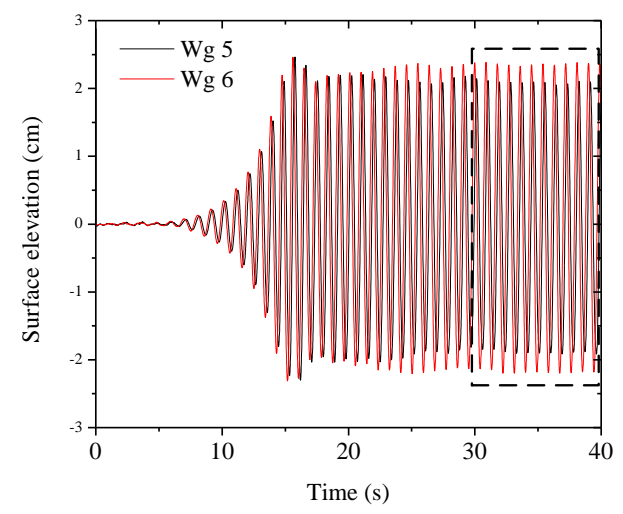

(a)

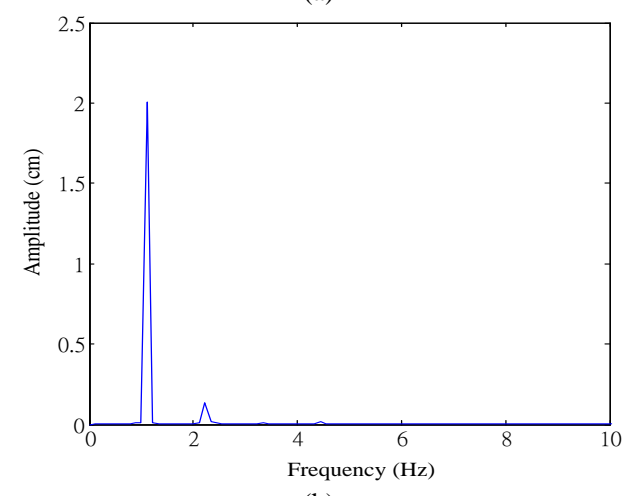

(b)

Fig. 2 (a) Time series and FFT result of surface elevations of (b) Wg 5 for Test F1.

A sample of the time series of actuator displacement for Test S1 is shown in Fig. 3a. Again the most stable sections of ten cycles (9 s, the section in the dotted frame) of displacement signals are chosen for FFT (the result is shown in Fig. 3b). The corresponding video recorded during this time section is digitized to obtain the wave runup. It should be noted that although efforts have been made to rigidly fix the frame holding the actuator on the flume wall, the actuator motion still induced high frequency components, as could be seen in the FFT of the actual actuator displacement (Fig. 3b). Although the amplitudes of such high frequency components are small $(<2 \%$ that of the fundamental frequency component), they could lead to similar high frequency components in the radiated wave and runup on the column.

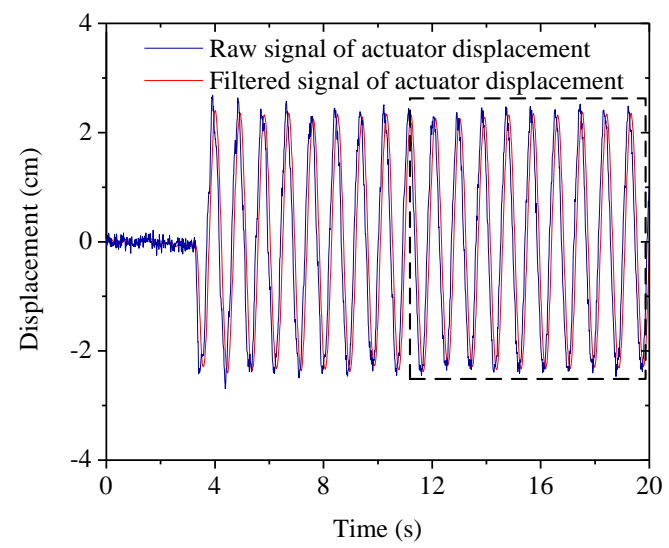

(a)

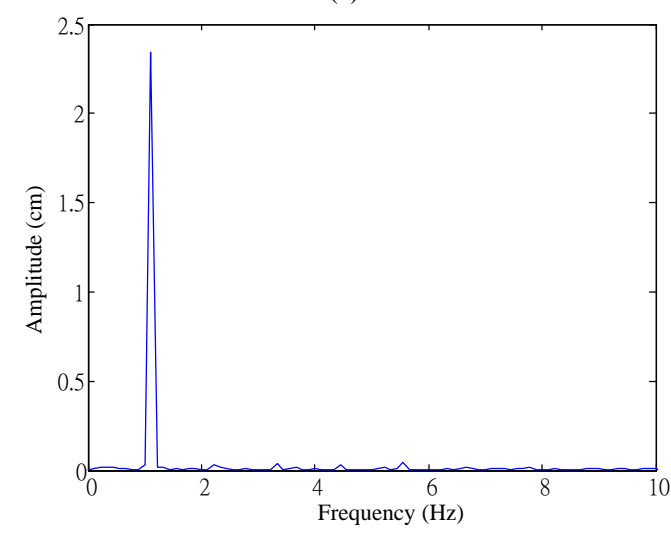

(b)

Fig. 3 (a) Time series and (b) FFT result of the actuator displacement signal for Test $\mathrm{S} 1$.

To remove the effects of high frequency components, the signals of $\mathrm{Wg} \mathrm{4}$, the velocity calculated based on the actual displacement signal and the runup profile, are filtered using a low pass filter (elliptic $2^{\text {nd }}$ order with cutoff frequency $=6$ ). Fig. 4 shows sample filtered signals of Test S1. The correlation between wave runup and surging velocity is then analyzed using these filtered signals.

As seen in Fig. 4, there are two peaks in the runup for each cycle of surge. There is also a minor phase difference between the velocity and runup. These two peaks are close to the trough and peak of actuator velocity which could be physically explained as follows. Firstly when the actuator velocity reaches a positive maximum value, the relative velocity between the cylinder and water particles surrounding it is similarly at a maximum, thus generating the first peak in the runup though with a possible phase difference. The second peak corresponds to when the 
actuator velocity reaches a negative maximum. Here the water particles behind the cylinder follow the cylinder velocity and thus its velocity will also increase negatively. After the cylinder velocity reaches its negative maximum value, it starts to decrease. Due to inertia, the water particles in front of the cylinder continue to run towards the cylinder, leading to the second runup peak, with another phase difference. Fig. 4 shows that the troughs of the runup occurs when the radiated wave surface $(\mathrm{Wg} 4)$ in front of the cylinder is in the trough while the surging velocity is zero, so that the relative velocity between the cylinder and water particles in front of it are zero.

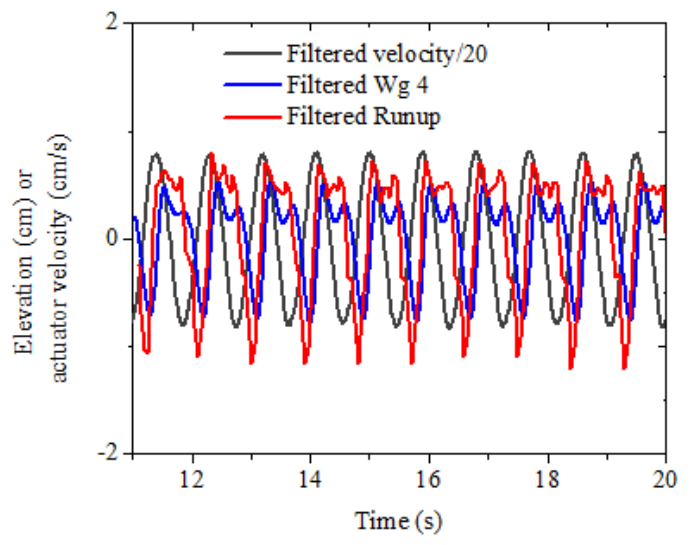

Fig. 4. Time series of wave runup, surface elevation of wave gauges 4 and filtered velocity for Test S1.

Based on the physical process presented above, the experimental data is analyzed using water particle velocities as listed in Table III. Linear wave theory is used to calculate the maximum horizontal water particle velocity at the wave amplitude.

TABLE III: EXTRACTED EXPERIMENTAL DATA FOR F1-F6 TEST

\begin{tabular}{llll}
\hline \hline Test No. & $\begin{array}{l}\eta \\
(\mathrm{cm})\end{array}$ & $u^{2} / 2 \mathrm{~g}(\mathrm{~cm})$ & $\begin{array}{l}R-\eta \\
(\mathrm{cm})\end{array}$ \\
\hline F1 & 2.16 & 0.143 & 0.638 \\
F2 & 2.49 & 0.099 & 0.410 \\
F3 & 2.38 & 0.056 & 0.218 \\
F4 & 2.29 & 0.035 & 0.108 \\
F5 & 2.35 & 0.030 & 0.049 \\
F6 & 3.53 & 0.069 & 0.369
\end{tabular}

Note: $R=$ runup on cylinder; $\eta=A_{w}$. The water particle velocities at the crest $u$ is calculated based on linear wave theory.

For cylinder surging in still water, the initial elevation $\eta$ is $0 \mathrm{~cm}$ (radiated wave only); $u$ is calculated to be based on the surge trajectory. For tests S1-S6, the velocity head $u^{2} / 2 \mathrm{~g}$ is calculated to be $0.136,0.088,0.057,0.044,0.043$ and 0.097 $\mathrm{cm}$, respectively; runup $(R-\eta)$ is calculated to be $0.8,0.5,0.3$, $0.2,0.15$ and $0.4 \mathrm{~cm}$, respectively.

Figs. 5 (a) and (b) show the plots of $(R-\eta)$ against velocity head $u^{2} / 2 \mathrm{~g}$. The difference is that the equation in Fig. $5 \mathrm{~b}$ is fitted with constraint that it must cross the origin while that in Fig. 5a hasn't such constraint. The constraint means that when velocity head is zero, $(R-\eta)=0$. It is noted $(R$ - crest $)$ is used to focus on the trend of runup and velocity head. For the fixed cylinder in wave, the runup is equal to the crest if water particle velocity head is neglected. In contrast, for the cylinder surging wave, the runup is equal to zero if surge velocity is zero.
Both figures show that the similar root mean square errors (rmse), thus based on the physical analysis, equation in Fig. $5 \mathrm{~b}$ is chosen. It shows that runup on cylinder increases linearly with the increasing velocity head as the velocity of wave water particle or surge motion increases. Although these two scenarios involve different physical mechanisms, the resulting runups are linearly correlated with the relative velocity between the cylinder and water particles around it.

The linear fitted equation in Fig. 5 (b) is

$$
R=\eta+4.7951 \frac{u^{2}}{2 g}
$$

It is apparent that Eq. (1) is similar with those listed in Table I, although the coefficient of the velocity head is different.

The above findings could have potential applications for the investigation of wave runup on a cylinder surging under incident waves, which is a combination of the two scenarios investigated in the current study. For such cases, the velocity should be the combined velocity of both surging cylinder and moving particles.

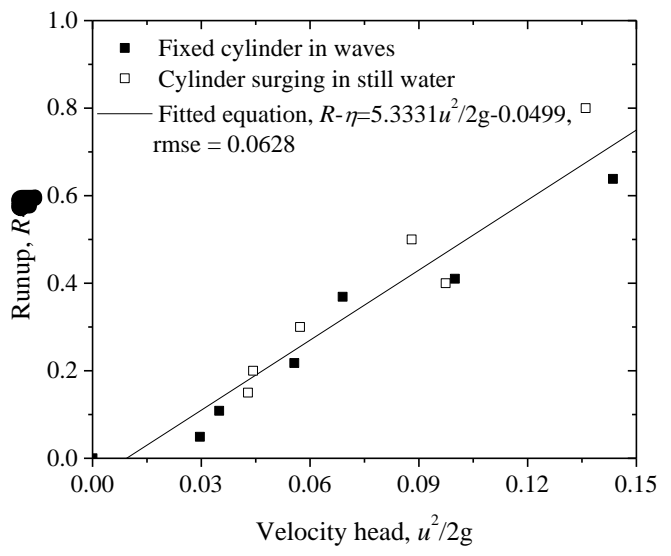

(a)

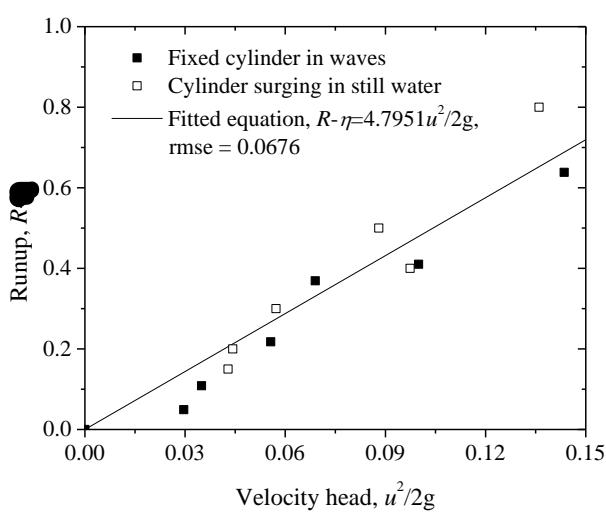

(b)

Fig. 5. Plot of runup against velocity head: (a)Fitted equation without constraint; (b)Fitted equation with constraint that the fitted line must cross the origin.

\section{CONCLUSIONS}

Experiments were conducted to investigate the relationship between wave runup on a cylinder and the relative velocity between the cylinder and water particles around it. Both the runup on a fixed cylinder in wave conditions and the runup on a surging cylinder in still water 
were studied. The results show that the runup is linearly increasing with increasing velocity head and can be fitted using the same linear relationship.

\section{ACKNOWLEDGMENT}

Support for the research from Singapore Maritime Institute Deepwater Technology (DWT) R\&D Programme is gratefully acknowledged. Dr. Hai Gu and Mr. Zhiping Cheng from American Bureau of Shipping (Singapore Innovation and Research Center), Dr. Xiaobo Chen and Dr. Binbin Li from Bureau Veritas (Singapore) and Dr. Chin Lee Lim from Sembcorp Marine Technology (Singapore), are acknowledged for valuable discussions at our project meetings.

\section{REFERENCES}

[1] R. J. Hallermeier, "Nonlinear flow of wave crests past a thin pile," Journal of Waterway, Harbours \& Coastal Engineering Division, vol. 102, no. 4, pp. 365-377, Nov. 1976.

[2] C. J. Galvin and R. J. Hallermeier, "Wave runup on vertical cylinders," Coastal Engineering Proceedings, vol. 1, no. 13, pp. 1-14, 1972.

[3] J. P. Haney and J. B. Herbich, "Wave flow around thin piles and pile groups," Journal of Hydraulic Research, vol. 20, no. 1, pp. 1-14, 1982.

[4] D. L. Kriebel, "Nonlinear wave interaction with a vertical circular cylinder. Part II: Wave run-up," Ocean Engineering, vol. 19, no. 1, pp. 75-99, Jan. 1992.

[5] J. M. Niedzwecki and A. Duggal, "Wave runup and forces on cylinders in regular and random waves," Journal of Waterway, Port, Coastal, and Ocean Engineering, vol. 118, no. 6, pp. 615-634, Nov. 1992.

[6] J. M. Niedzwecki and J. R. Huston, "Wave interaction with tension leg platforms," Ocean Engineering, vol. 19, no. 1, pp. 21-37, Jan. 1992.

[7] A. Martin, W. Easson, and T. Bruce, "Runup on columns in steep, deep water regular waves," Journal of Waterway, Port, Coastal, and Ocean Engineering, vol. 127, no. 1, pp. 26-32, 2001.

[8] L. De Vos, P. Frigaard, and J. De Rouck, "Wave runup on cylindrical and cone shaped foundations for offshore wind turbines," Coastal Engineering, vol. 54, no. 1, pp. 17-29, Jan. 2007.

[9] T. Lykke Andersen, P. Frigaard, M. L. Damsgaard, and L. De Vos, "Wave run-up on slender piles in design conditions - Model tests and design rules for offshore wind," Coastal Engineering, vol. 58, no. 4, pp 281-289, Apr. 2011.

[10] J. Ramirez, P. Frigaard, T. L. Andersen, and L. De Vos, "Large scale model test investigation on wave run-up in irregular waves at slender piles," Coastal Engineering, vol. 72, pp. 69-79, Feb. 2013.

[11] Y. Goda and T. Suzuki, "Estimation of incident and reflected waves in random wave. Experiments," in Proc. 15th Conference on Coastal Engineering, Honolulu, Hawaii, USA, 1976.

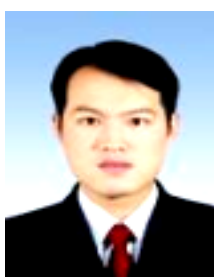

Engineering.

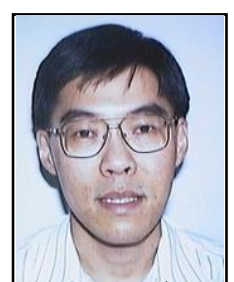

Edmond Lo Yat-Man received his doctor's degree in civil engineering (hydrodynamics) from Massachusetts Institute of Technology in 1985.

$\mathrm{He}$ is an associate professor in School of Civil and Environmental Engineering, Nanyang Technological University.

Prof. Lo joined NTU in 1996 where he was the head of the Division of Environmental and Water Resources Engineering (2005-08) and the chair of the School of Civil and Environmental Engineering (2008-2011). He is also currently the deputy director of NTU's Institute of Catastrophe Risk Management and the dep. director of the Maritime Institute@NTU.

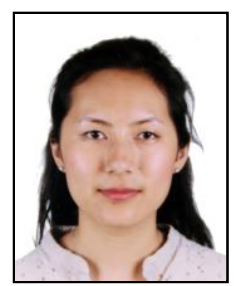

Wei Jian is a research fellow at Maritime Institute at Nanyang Technological University, Singapore.

She received her bachelor of engineering degree from University of Canterbury, New Zealand in 2008 and $\mathrm{Ph} . \mathrm{D}$ in engineering from University of Cambridge, UK in 2013.

Dr. Jian's current research interest includes computational fluid dynamics application in offshore structures.

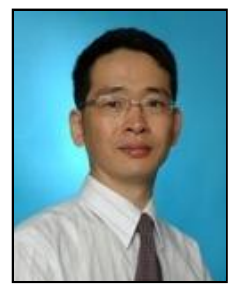

Zhenhua Huang obtained his doctor's degree in environmental fluid mechanics from Massachusetts Institute of Technology in 2004.

$\mathrm{He}$ is an associate professor in Ocean and Resources Engineering Department, School of Ocean and Earth Science and Technology, University of Hawaii.

Prof. Huang is a member of American Geophysical Union (AGU), Member of International Association for Hydro-Environment Engineering (IAHR), Member of American Coast and Education Society (CERF) 